\title{
Multiscale Analysis of Composite Structures
}

\author{
Claudia Timofte \\ Faculty of Physics \\ University of Bucharest, Bucharest, Romania \\ Email: claudiatimofte@yahoo.com
}

Received: 15 July 2012, accepted: 2 September 2012, published: 9 October 2012

\begin{abstract}
The goal of this paper is to present some homogenization results for diffusion problems in composite structures, formed by two media with different features. Our setting is relevant for modeling heat diffusion in composite materials with imperfect interfaces or electrical conduction in biological tissues. The approach we follow is based on the periodic unfolding method, which allows us to deal with general media.
\end{abstract}

Keywords-homogenization; the periodic unfolding method; dynamical boundary condition

\section{Introduction AND Setting of the Problem}

The analysis of diffusion phenomena in highly heterogeneous materials has been a subject of huge interest in the last decades. The purpose of this paper is to analyze the effective behavior of the solution of some nonlinear problems arising in the modeling of diffusion in a periodic structure formed by two media with different properties, separated by an active interface. Our setting is relevant for modeling heat conduction in composite materials with imperfect interfaces or electrical conduction in biological tissues. We assume first that both components are connected. Using the periodic unfolding method, which allows us to deal with general heterogeneous media, we can describe the evolution in time of the homogenized solution. The model we obtain at the macroscale is a bidomain model, which conceives the composite material, despite of its discrete structure, as the coupling of two continuous superimposed domains. Our model is a generalization of the so-called Barenblatt model, arising in the context of diffusion in partially fissured media. A similar model appears also in the study of the bioelectrical activity of the heart at a macroscopic level. In this case, at the microscopic scale, we deal with a medium composed of two different conductive phases (the intracellular and extracellular spaces), separated by a dielectric interface (the cellular membranes), which has a capacitive and a nonlinear conductive behavior. The electric potential verifies elliptic equations in the two conductive regions, coupled by a suitable evolutive boundary condition involving the potential jump at the interfaces between the two phases. The evolution in time of the homogenized potential is governed exactly by a bidomain model. We shall also briefly discuss a different geometric situation, in which only one phase is connected, while the other one is disconnected. In this case, we are led at a different macroscopic model.

Let $\Omega$ be a bounded domain in $\mathbb{R}^{n}(n \geq 3)$, with a Lipschitz boundary $\partial \Omega$ consisting of a finite number of connected components. We consider the case in which $\Omega$ is a periodic structure formed by two components, $\Omega^{\varepsilon}$ and $\Pi^{\varepsilon}$, representing two materials with different features, separated by an interface $S^{\varepsilon}$. We assume that both $\Omega^{\varepsilon}$ and $\Pi^{\varepsilon}=\Omega \backslash \overline{\Omega^{\varepsilon}}$ are connected, but only $\Omega^{\varepsilon}$ reaches the external fixed boundary of the domain $\Omega$. Here, $\varepsilon$ represents a small parameter related to the characteristic size of the our two regions. More precisely, let $Y_{1}$ be a Lipschitz open connected subset of the unit cell $Y=(0,1)^{n}$. Let $Y_{2}=Y \backslash \overline{Y_{1}}$. We suppose that $Y_{2}$ has a locally Lipschitz boundary $\Gamma$ and we assume that the intersections of the boundary of $Y_{2}$ with the boundary of $Y$ are identically reproduced on opposite faces of the cell, which are denoted, for any $1 \leq i \leq n$, by

$$
\begin{aligned}
\Sigma_{i} & =\left\{y \in \partial Y \mid y_{i}=1\right\}, \\
\Sigma_{-i} & =\left\{y \in \partial Y \mid y_{i}=0\right\} .
\end{aligned}
$$


We suppose that repeating $Y$ by periodicity, the union of all the sets $\overline{Y_{1}}$ is connected and has a locally $C^{2}$ boundary. Also, we assume that the origin of the coordinate system is set in a ball contained in this union (see [8]).

Let

$$
\begin{gathered}
Z_{\varepsilon}=\left\{k \in \mathbb{Z}^{n} \mid \varepsilon k+\varepsilon Y \subseteq \Omega\right\}, \\
K_{\varepsilon}=\left\{k \in Z_{\varepsilon} \mid \varepsilon k \pm \varepsilon e_{i}+\varepsilon Y \subseteq \Omega, \forall i=\overline{1, n}\right\},
\end{gathered}
$$

where $e_{i}$ are the elements of the canonical basis of $\mathbb{R}^{n}$. We define

$$
\Pi^{\varepsilon}=\operatorname{int}\left(\bigcup_{k \in K_{\varepsilon}}\left(\varepsilon k+\varepsilon \overline{Y_{2}}\right)\right)
$$

and

$$
\Omega^{\varepsilon}=\Omega \backslash \overline{\Pi^{\varepsilon}}
$$

and we set $\theta=\left|Y \backslash \overline{Y_{2}}\right|$.

Let $\alpha_{1}, \beta_{1} \in \mathbb{R}$ such that $0<\alpha_{1}<\beta_{1}$. We denote by $\mathcal{M}\left(\alpha_{1}, \beta_{1}, Y\right)$ the set of all the square matrices $A \in\left(L^{\infty}(Y)\right)^{n \times n}$ such that, for any $\xi \in \mathbb{R}^{n}$, we have $(A(y) \xi, \xi) \geq \alpha_{1}|\xi|^{2},|A(y) \xi| \leq \beta_{1}|\xi|$, almost everywhere in $Y$. We consider a family of matrices $A^{\varepsilon}(x)=A\left(\frac{x}{\varepsilon}\right)$ defined on $\Omega$, where $A \in \mathcal{M}\left(\alpha_{1}, \beta_{1}, Y\right)$ is a symmetric smooth $Y$-periodic matrix. We shall denote the matrix $A$ by $A_{1}$ in $Y_{1}$ and by $A_{2}$, respectively, in $Y_{2}$.

If $(0, T)$ is the time interval, we shall analyze the macroscopic behavior of the solutions of the following system:

$$
\left\{\begin{array}{l}
-\operatorname{div}\left(A_{1}^{\varepsilon} \nabla u^{\varepsilon}\right)+\beta\left(u^{\varepsilon}\right)=f \quad \text { in } \Omega^{\varepsilon} \times(0, T), \\
-\operatorname{div}\left(A_{2}^{\varepsilon} \nabla v^{\varepsilon}\right)=f \text { in } \Pi^{\varepsilon} \times(0, T), \\
A_{1}^{\varepsilon} \nabla u^{\varepsilon} \cdot \nu=A_{2}^{\varepsilon} \nabla v^{\varepsilon} \cdot \nu \text { on } S^{\varepsilon} \times(0, T), \\
A_{1}^{\varepsilon} \nabla u^{\varepsilon} \cdot \nu+\alpha \varepsilon \frac{\partial}{\partial t}\left(u^{\varepsilon}-v^{\varepsilon}\right)= \\
a \varepsilon g\left(v^{\varepsilon}-u^{\varepsilon}\right) \text { on } S^{\varepsilon} \times(0, T), \\
u^{\varepsilon}=0 \text { on } \partial \Omega \times(0, T), \\
u^{\varepsilon}(0, x)-v^{\varepsilon}(0, x)=c^{0}(x) \text { on } S^{\varepsilon} .
\end{array}\right.
$$

Here, $\nu$ is the unit outward normal to $\Omega^{\varepsilon}, a>0, f \in$ $L^{2}\left(0, T ; L^{2}(\Omega)\right), c^{0} \in H_{0}^{1}(\Omega), \alpha>0$ and $\beta$ and $g$ are continuous functions, monotonously non-decreasing and such that $\beta(0)=0$ and $g(0)=0$. We shall suppose that there exist a positive constant $C$ and an exponent $q$, with $0 \leq q \leq n /(n-2)$, such that

$$
|\beta(v)| \leq C\left(1+|v|^{q}\right), \quad|g(v)| \leq C\left(1+|v|^{q}\right) .
$$

As examples of such functions, we mention the case of Langmuir or Freundlich kinetics. For the case of electrical conduction in biological tissues, we may consider that $f=0, \beta=0$ and $g$ is, in $\mathbb{R}^{3}$, a cubic function, like in the Fitzhugh-Nagumo model (see, for instance, [9]).
Results concerning the well posedness of problem (1) in suitable function spaces and proper energy estimates were obtained in [1], [3] and [9].

Using the periodic unfolding method recently introduced by D. Cioranescu, A. Damlamian, G. Griso, P. Donato and R. Zaki (see [4] and [5]), we can prove that the asymptotic behavior of the solution of our problem is governed by a new nonlinear system (see (3)). At a macroscopic level, the composite material can be represented by a continuous model, which describes it as the superimposition of two interpenetrating continuous media, coexisting at every point of the domain. Our macroscopic model is a degenerate parabolic system, as the time derivatives involve the unknown $v-u$.

If we deal with a different geometry, i.e. we consider that only one phase is connected, while the other one is disconnected, we are led to a different macroscopic model (see Remark 2.).

Similar problems have been considered, using different techniques, in [1] and [9], for studying electrical conduction in biological tissues.

The results presented in this paper constitute a generalization of those obtained in [2], [8] and [10].

As already mentioned, our approach is based on the periodic unfolding method, which allows us to deal with general media (see Remark 3.). For dealing with such two-component domains, we use unfolding operators, which map functions defined on oscillating domains into functions defined on fixed domains. In such a way, we can avoid the use of extension operators. Therefore, using this general method, we can deal with media with less regularity than those usually considered in the literature (composite materials and biological tissues are highly heterogeneous and their interfaces are not very smooth, in general).

The plan of the paper is as follows: in the second section, we give the main convergence result of this paper. The last section is devoted to the proof of our result.

\section{The MAIN Result}

Using the periodic unfolding method, we can pass to the limit in the variational formulation of problem (1) and we obtain the effective behavior of the solution of our microscopic model.

Theorem 1. The solution $\left(u^{\varepsilon}, v^{\varepsilon}\right)$ of system (1) converges, as $\varepsilon \rightarrow 0$, to the unique solution $(u, v)$, with $u, v \in L^{2}\left(0, T ; H_{0}^{1}(\Omega)\right), \frac{\partial}{\partial t}(u-v) \in L^{2}\left(0, T ; L^{2}(\Omega)\right)$ 
and $u, v \in C^{0}\left([0, T] ; H_{0}^{1}(\Omega)\right)$, of the following macroscopic problem:

$$
\left\{\begin{array}{l}
\alpha|\Gamma| \frac{\partial}{\partial t}(u-v)-\operatorname{div}\left(\bar{A}^{1} \nabla u\right)+\theta \beta(u)- \\
a|\Gamma| g(v-u)=\theta f \text { in } \Omega \times(0, T), \\
\alpha|\Gamma| \frac{\partial}{\partial t}(v-u)-\operatorname{div}\left(\bar{A}^{2} \nabla v\right)+ \\
a|\Gamma| g(v-u)=(1-\theta) f \text { in } \Omega \times(0, T), \\
u(0, x)-v(0, x)=c^{0}(x) \text { on } \Omega .
\end{array}\right.
$$

In (3), $\bar{A}^{1}$ and $\bar{A}^{2}$ are the homogenized matrices, defined by:

$$
\begin{aligned}
\bar{A}_{i j}^{1} & =\int_{Y_{1}}\left(a_{i j}+a_{i k} \frac{\partial \chi_{1 j}}{\partial y_{k}}\right) d y, \\
\bar{A}_{i j}^{2} & =\int_{Y_{2}}\left(a_{i j}+a_{i k} \frac{\partial \chi_{2 j}}{\partial y_{k}}\right) d y
\end{aligned}
$$

and $\chi_{1 k} \in H_{p e r}^{1}\left(Y_{1}\right) / \mathbb{R}, \chi_{2 k} \in H_{p e r}^{1}\left(Y_{2}\right) / \mathbb{R}, k=$ $1, \ldots, n$, are the weak solutions of the cell problems

$$
\begin{aligned}
& \left\{\begin{array}{l}
-\nabla_{y} \cdot\left(\left(A_{1}(y) \nabla_{y} \chi_{1 k}\right)=\nabla_{y} A_{1}(y) e_{k}, \quad y \in Y_{1},\right. \\
\left(A_{1}(y) \nabla_{y} \chi_{1 k}\right) \cdot \nu=-A_{1}(y) e_{k} \cdot \nu, \quad y \in \Gamma,
\end{array}\right. \\
& \left\{\begin{array}{l}
-\nabla_{y} \cdot\left(\left(A_{2}(y) \nabla_{y} \chi_{2 k}\right)=\nabla_{y} A_{2}(y) e_{k}, \quad y \in Y_{2},\right. \\
\left(A_{2}(y) \nabla_{y} \chi_{2 k}\right) \cdot \nu=-A_{2}(y) e_{k} \cdot \nu, \quad y \in \Gamma .
\end{array}\right.
\end{aligned}
$$

So, at a macroscopic scale, we obtain a new system, which is similar to the bidomain model, appearing in the context of diffusion in partially fissured media or in the context of electrical activity of the heart (for this case, $f=0, \beta=0$ ).

Remark 2. If we consider the case of a different geometry, i.e. if we assume that $\Omega^{\varepsilon}$ is still connected, but $\Pi^{\varepsilon}$ is disconnected, then the homogenized matrix $\bar{A}^{2}=0$ and system (3) consists in the coupling of a partial differential equation and an ordinary differential one.

\section{Proof of the Main Result}

We shall only sketch the proof of our main convergence result. For details, we refer to [11]. (1):

Let us consider the variational formulation of problem

$$
\begin{gathered}
\int_{0}^{T} \int_{\Omega^{\varepsilon}} A_{1}^{\varepsilon} \nabla u^{\varepsilon} \cdot \nabla \varphi d x d t+\int_{0}^{T} \int_{\Pi^{\varepsilon}} A_{2}^{\varepsilon} \nabla v^{\varepsilon} \cdot \nabla \varphi d x d t+ \\
\int_{0}^{T} \int_{\Omega^{\varepsilon}} \beta\left(u^{\varepsilon}\right) \varphi d x d t+\alpha \varepsilon \int_{0}^{T} \int_{S^{\varepsilon}}\left(u^{\varepsilon}-v^{\varepsilon}\right) \frac{\partial}{\partial t}[\varphi] d \sigma d t+ \\
\alpha \varepsilon \int_{S^{\varepsilon}}\left(u^{\varepsilon}-v^{\varepsilon}\right)(0)[\varphi](0) d \sigma+
\end{gathered}
$$

$$
a \varepsilon \int_{0}^{T} \int_{S^{\varepsilon}} g\left(v^{\varepsilon}-u^{\varepsilon}\right)[\varphi] d \sigma d t=\int_{0}^{T} \int_{\Omega} f \varphi d x d t
$$

for any $\varphi \in L^{2}(\Omega \times(0, T))$ such that

$$
\begin{gathered}
\varphi_{\left.\right|_{\Omega^{\varepsilon}}} \in L^{2}\left(0, T ; H^{1}\left(\Omega^{\varepsilon}\right)\right), \quad \varphi_{\left.\right|_{\Pi^{\varepsilon}}} \in L^{2}\left(0, T ; H^{1}\left(\Pi^{\varepsilon}\right)\right), \\
{[\varphi] \in H^{1}\left(0, T ; L^{2}\left(S^{\varepsilon}\right)\right),}
\end{gathered}
$$

$\varphi$ vanishes on $\partial \Omega \times(0, T)$ and $\varphi$ vanishes at $t=T$. Here, we have denoted by $[\varphi]$ the difference of the traces of $\varphi_{\left.\right|_{\Omega \varepsilon}}$ and $\varphi_{\left.\right|_{\Pi \varepsilon}}$ on $S^{\varepsilon}$.

There exists a unique weak solution $\left(u^{\varepsilon}, v^{\varepsilon}\right)$ of (4), with $u^{\varepsilon} \in L^{2}\left(0, T ; H_{\partial \Omega}^{1}\left(\Omega^{\varepsilon}\right)\right), v^{\varepsilon} \in L^{2}\left(0, T ; H^{1}\left(\Pi^{\varepsilon}\right)\right)$, where

$$
H_{\partial \Omega}^{1}\left(\Omega^{\varepsilon}\right)=\left\{u \in H^{1}\left(\Omega^{\varepsilon}\right) \mid u=0 \text { on } \partial \Omega \cap \partial \Omega^{\varepsilon}\right\} .
$$

Under the above hypotheses on the data, we can obtain suitable a priori estimates, independent of $\varepsilon$, for our solution (see [6], [9] and [10]):

$$
\begin{gathered}
\int_{0}^{t} \int_{\Omega^{\varepsilon}} A_{1}^{\varepsilon} \nabla u^{\varepsilon} \cdot \nabla \varphi d x d t+\int_{0}^{t} \int_{\Pi^{\varepsilon}} A_{2}^{\varepsilon} \nabla v^{\varepsilon} \cdot \nabla \varphi d x d t+ \\
\varepsilon \int_{S^{\varepsilon}}\left(u^{\varepsilon}-v^{\varepsilon}\right)^{2} d \sigma \leq C,
\end{gathered}
$$

where $0<t<T$ and $C$ is independent of $\varepsilon$.

For dealing with such two-component domains, we use two unfolding operators, $\mathcal{T}_{1}^{\varepsilon}$ and $\mathcal{T}_{2}^{\varepsilon}$, which map functions defined on the oscillating domains into functions defined on fixed domains. In such a way, we can avoid the use of extension operators (see [4] and [7]). Also, we shall make use of the boundary unfolding operator, $\mathcal{T}_{b}^{\varepsilon}$, introduced in [5]. Therefore, using the above mentioned unfolding operators, we can prove that there exist $u, v \in L^{2}\left(0, T ; H_{0}^{1}(\Omega)\right), \widehat{u} \in L^{2}((0, T) \times$ $\left.\Omega ; H_{p e r}^{1}\left(Y_{1}\right)\right), \widehat{v} \in L^{2}\left((0, T) \times \Omega ; H_{\text {per }}^{1}\left(Y_{2}\right)\right)$ such that, up to a subsequence, for $\varepsilon \rightarrow 0$, we have:

$$
\mathcal{T}_{1}^{\varepsilon}\left(u^{\varepsilon}\right) \rightarrow u \quad \text { strongly in } L^{2}\left((0, T) \times \Omega, H^{1}\left(Y_{1}\right)\right),
$$

$\mathcal{T}_{1}^{\varepsilon}\left(\nabla u^{\varepsilon}\right) \rightarrow \nabla u+\nabla_{y} \widehat{u} \quad$ weakly in $L^{2}\left((0, T) \times \Omega \times Y_{1}\right)$,

$$
\mathcal{T}_{2}^{\varepsilon}\left(v^{\varepsilon}\right) \rightarrow v \quad \text { strongly in } L^{2}\left((0, T) \times \Omega, H^{1}\left(Y_{2}\right)\right),
$$

$\mathcal{T}_{2}^{\varepsilon}\left(\nabla v^{\varepsilon}\right) \rightarrow \nabla v+\nabla_{y} \widehat{v} \quad$ weakly in $L^{2}\left((0, T) \times \Omega \times Y_{2}\right)$.

Moreover,

$$
\frac{\partial}{\partial t}(u-v) \in L^{2}\left(0, T ; L^{2}(\Omega)\right)
$$

and 
In order to obtain the limit problem (3), we take, in a first step, $\Phi_{1}, \Phi_{2} \in C_{0}^{\infty}(\Omega)=\mathcal{D}(\Omega)$ and $\Psi \in$ $C_{0}^{\infty}((0, T))=\mathcal{D}(0, T)$. We have:

$$
\begin{gathered}
\int_{0}^{T} \int_{\Omega^{\varepsilon}} A_{1}^{\varepsilon} \nabla u^{\varepsilon} \cdot \nabla \Phi_{1} \Psi d x d t+ \\
\int_{0}^{T} \int_{\Pi^{\varepsilon}} A_{2}^{\varepsilon} \nabla v^{\varepsilon} \cdot \nabla \Phi_{2} \Psi d x d t+\int_{0}^{T} \int_{\Omega^{\varepsilon}} \beta\left(u^{\varepsilon}\right) \Phi_{1} \Psi d x d t \\
+\alpha \varepsilon \int_{0}^{T} \int_{S^{\varepsilon}}\left(u^{\varepsilon}-v^{\varepsilon}\right)\left(\Phi_{2}-\Phi_{1}\right) \frac{d \Psi}{d t} d \sigma d t+ \\
a \varepsilon \int_{0}^{T} \int_{S^{\varepsilon}} g\left(u^{\varepsilon}-v^{\varepsilon}\right)\left(\Phi_{2}-\Phi_{1}\right) \Psi d \sigma d t= \\
\int_{0}^{T} \int_{\Omega^{\varepsilon}} f \Phi_{1} \Psi d x d t+\int_{0}^{T} \int_{\Pi^{\varepsilon}} f \Phi_{2} \Psi d x d t
\end{gathered}
$$

Applying the corresponding unfolding operators in (5) and passing to the limit, with $\varepsilon \rightarrow 0$, we get (see, for details, [4], [6], [10] and [11]):

$$
\begin{gathered}
\int_{0}^{T} \int_{\Omega \times Y_{1}} A_{1}\left(\nabla u+\nabla_{y} \widehat{u}\right) \cdot \nabla \Phi_{1} \Psi d x d y d t+ \\
\int_{0}^{T} \int_{\Omega \times Y_{2}} A_{2}\left(\nabla v+\nabla_{y} \widehat{v}\right) \cdot \nabla \Phi_{2} \Psi d x d y d t+ \\
\int_{0}^{T} \int_{\Omega \times Y_{1}} \beta(u) \Phi_{1} \Psi d x d y d t+ \\
\alpha \int_{0}^{T} \int_{\Omega \times \Gamma}(u-v)\left(\Phi_{2}-\Phi_{1}\right) \frac{d \Psi}{d t} d x d \sigma d t+ \\
a \int_{0}^{T} \int_{\Omega \times \Gamma} g(u-v)\left(\Phi_{2}-\Phi_{1}\right) \Psi d x d \sigma d t= \\
\int_{0}^{T} \int_{\Omega \times Y_{1}} f \Phi_{1} \Psi d x d y d t+ \\
\int_{0}^{T} \int_{\Omega \times Y_{2}} f \Phi_{2} \Psi d x d y d t .
\end{gathered}
$$

In a second step, we take the test functions $w_{i}^{\varepsilon}=$ $\varepsilon \Phi_{i}(x) \varphi_{i}\left(\frac{x}{\varepsilon}\right) \Psi(t)$, with $i=1,2$, where $\Phi \in \mathcal{D}(\Omega), \varphi_{i} \in$ $H_{\text {per }}^{1}\left(Y_{i}\right), \stackrel{\varepsilon}{\Psi} \in \mathcal{D}((0, T))$. Observing that $\mathcal{T}_{i}^{\varepsilon}\left(w_{i}^{\varepsilon}\right) \rightarrow 0$ strongly in $L^{2}\left((0, T) \times \Omega \times Y_{i}\right)$ and $\mathcal{T}_{i}^{\varepsilon}\left(\nabla w_{i}^{\varepsilon}\right) \rightarrow \Phi_{i} \nabla_{y} \varphi_{i}$, strongly in $L^{2}\left((0, T) \times \Omega \times Y_{i}\right)$, we can pass to the limit and we get:

$$
\int_{0}^{T} \int_{\Omega \times Y_{1}} A_{1}\left(\nabla u+\nabla_{y} \widehat{u}\right) \cdot \nabla_{y} \varphi_{1} \Phi_{1} \Psi d x d y d t+
$$

$$
\int_{0}^{T} \int_{\Omega \times Y_{2}} A_{2}\left(\nabla v+\nabla_{y} \widehat{v}\right) \cdot \nabla_{y} \varphi_{2} \Phi_{2} \Psi d x d y d t=0 .
$$

Putting together (6) and (7) and using standard density arguments, we obtain exactly the variational formulation of the limit problem (3). We can easily pass to the limit, with $\varepsilon \rightarrow 0$, in the initial condition and we obtain $u(0, x)-v(0, x)=c^{0}(x), \forall x \in \Omega$.

As $u$ and $v$ are uniquely determined (see [9]), the whole sequences of microscopic solutions converge to a solution of the unfolded limit problem and this completes the proof of Theorem 1.

Remark. 3 The above results can be extended to the case in which $A^{\varepsilon}$ is a sequence of matrices in $\mathcal{M}\left(\alpha_{1}, \beta_{1}, \Omega\right)$ such that

$$
\mathcal{T}_{i}^{\varepsilon}\left(A^{\varepsilon}\right) \rightarrow A \quad \text { a.e. in } \Omega \times Y,
$$

with $i=1,2$ and $A=A(x, y) \in \mathcal{M}\left(\alpha_{1}, \beta_{1}, \Omega \times Y\right)$. The only difference is that in this case the homogenized matrices are no longer constant and depend on $x$.

\section{CONCLUSION}

Using the periodic unfolding method, the effective behavior of the solution of some problems arising in the modeling of diffusion processes in a periodic structure formed by two media with different properties, separated by an active interface, was analyzed. Two interesting geometric situations were discussed, leading to different macroscopic models. Our setting is relevant for studying the heat conduction in composite materials with imperfect interfaces or the electrical conduction in biological tissues.

\section{REFERENCES}

[1] M. Amar, D. Andreucci, P. Bisegna and R. Gianni,"On a hierarchy of models for electrical conduction in biological tissues", Math. Meth. Appl. Sci., vol. 29 no. 7, pp. 767-787, 2006. http://dx.doi.org/10.1002/mma.709

[2] G. I. Barenblatt, Y. P. Zheltov and I. N. Kochina, "Basic concepts in the theory of seepage of homogeneous liquids in fissured rocks (strata)", Prikl. Mat. Mekh., vol. 24, pp. 852-864, 1960.

[3] H. Brézis, "Problèmes unilatéraux", J. Math. Pures et Appl., vol. 51 no. 1, pp. 1-168, 1972.

[4] D. Cioranescu, A. Damlamian and G. Griso, "The periodic unfolding method in homogenization", SIAM J. Math. Anal., vol. 40 no. 4, pp. 1585-1620, 2008. http://dx.doi.org/10.1137/080713148

[5] D. Cioranescu, P. Donato and R. Zaki, "Asymptotic behavior of elliptic problems in perforated domains with nonlinear boundary conditions", Asymptotic Anal., vol. 53 no. 4, pp. 209-235, 2007.

[6] C. Conca, J. I. Díaz and C. Timofte, "Effective chemical processes in porous media", Math. Models Methods Appl. Sci. (M3AS), vol. 13 (10), pp. 1437-1462 (2003). 
[7] P. Donato, K. H. Le Nguyen, and R. Tardieu, "The periodic unfolding method for a class of imperfect transmission problems", Journal of Mathematical Sciences, vol. 176 no. 6, pp. 891-927, 2011.

http://dx.doi.org/10.1007/s10958-011-0443-2

[8] H. I. Ene and D. Polisevski, "Model of diffusion in partially fissured media", Z. Angew. Math. Phys., vol. 53 no. 6, pp. 1052 1059, 2002.

http://dx.doi.org/10.1007/PL00013849

[9] M. Pennacchio, G. Savaré and P. C. Franzone, "Multiscale modeling for the bioelectric activity of the heart", SIAM J. Math. Anal., vol. 37 no. 4, pp. 1333-1370, 2005. http://dx.doi.org/10.1137/040615249

[10] C. Timofte, "Multiscale analysis in nonlinear thermal diffusion problems in composite structures", Central Eur. J. Physics, vol. 8 no. 4 , pp. $555-561,2010$.

http://dx.doi.org/10.2478/s11534-009-0141-6

[11] C. Timofte, "Multiscale analysis of diffusion processes in composite media", in preparation, 2012.

[12] M. Veneroni, "Reaction-Diffusion systems for the microscopic cellular model of the cardiac electric field", Math. Meth. Appl. Sci., vol. 29 no. 14, pp. 1631-1661, 2006.

http://dx.doi.org/10.1002/mma.740 